ARTICLE

DOI: $10.1038 /$ s41467-017-00685-3

OPEN

\title{
Soft material for soft actuators
}

\author{
Aslan Miriyev ${ }^{1}$, Kenneth Stack $^{1} \&$ Hod Lipson (1) $^{1}$
}

Inspired by natural muscle, a key challenge in soft robotics is to develop self-contained electrically driven soft actuators with high strain density. Various characteristics of existing technologies, such as the high voltages required to trigger electroactive polymers ( $>1 \mathrm{KV}$ ), low strain $(<10 \%)$ of shape memory alloys and the need for external compressors and pressure-regulating components for hydraulic or pneumatic fluidicelastomer actuators, limit their practicality for untethered applications. Here we show a single self-contained soft robust composite material that combines the elastic properties of a polymeric matrix and the extreme volume change accompanying liquid-vapor transition. The material combines a high strain (up to 900\%) and correspondingly high stress (up to $1.3 \mathrm{MPa}$ ) with low density (0.84 $\mathrm{g} \mathrm{cm}^{-3}$ ). Along with its extremely low cost (about 3 cent per gram), simplicity of fabrication and environment-friendliness, these properties could enable new kinds of electrically driven entirely soft robots.

\footnotetext{
${ }^{1}$ Department of Mechanical Engineering, Columbia University in the City of New York, 500W 120th St., Mudd 220, New York, NY 10027, USA. Correspondence and requests for materials should be addressed to H.L. (email: hod.lipson@columbia.edu)
} 
nspired by biology, researchers aim to develop soft-bodied programmable motion in order to combine natural compliance with controllable actuation. One of the long standing challenges has been the lack of easily processed robust soft actuators with high strain density ${ }^{1-5}$. Such actuators would be easy to produce and to mold, cut, and $3 \mathrm{D}$ print into a desired shape, yet would produce large macroscopic actuation at relatively low voltage and current. Today, soft actuation techniques are based on either electroactive polymers ${ }^{6-12}$, shape memory alloys and shape memory polymers ${ }^{13-15}$, or compressed air and pressurized fluids actuators ${ }^{16-24}$. However, the high voltages required to trigger electroactive polymers $(>1 \mathrm{KV})$ and low strain $(<10 \%)$ of shape memory alloys, as well as the need for external compressors and pressure-regulating components for hydraulic ${ }^{16}$, $18,21,24$ or pneumatic 16, 17, 19,20,22,23 fluidic elastomer actuators, limit their miniaturization ${ }^{2,4}, 16$ and practicality for untethered applications. Recent demonstrations of actuation based on combustion $^{25}$ are ideal for impact delivery, but are less suitable for controllable kinematics.

Phase change materials offer an attractive alternative to conventional electromechanical actuators. Such materials rely on the mechanical force produced by the rapid expansion that occurs at the phase transition temperature. One of the classic examples of phase change materials is paraffin, which thermomechanical properties were first utilized in early $1930 \mathrm{~s}^{26}$ for self-regulating vents in greenhouses. While paraffin-based actuators can deliver large forces, their strain remains in the order of $10 \%$ volumetric change ${ }^{26-28}$, a strain that is on par with shape memory alloys and too small for most robotics applications.

A significantly higher expansion strain may be achieved by utilizing reversible liquid-vapor phase transition, but such material systems have been traditionally difficult to contain and control. A number of recent devices use entrapped liquid inside balloons or between thin films, to form expanding cavities ${ }^{29-32}$. Electrically triggered deformation of soft elastomer membranes, utilizing liquid-gas transition of liquid, was reported to show large area expansion ${ }^{33}$. However, such devices are challenging to manufacture and to form into arbitrary shapes because of their intricate internal design. For example, it is difficult to directly cast or $3 \mathrm{D}$-print any of these actuators.

Here we propose a single easily prepared soft robust material that combines the elastic properties of a polymeric matrix and the extreme volume change of a fluid upon liquid-vapor transition. We show and characterize the soft composite material comprised of a silicone elastomer matrix with ethanol distributed throughout it in micro-bubbles, exhibiting strains up to $900 \%$, and demonstrate its use as an actuator in a range of robotic applications.

\section{Results}

Materials system and its principles of action. Choosing a polymer matrix and a fluid for the composite meta-material system was guided by the desired mechanical properties of a polymer, boiling point and practical handling restrictions of a fluid, and chemical compatibility of the two. We aimed to synthesize a cheap, simple, user- and environment-friendly material comprised of food-safe and bio-compatible materials. We chose PDMS-based silicone elastomer, a non-hazardous elastomer widely used for soft robotic applications, as a matrix material, and ethanol, a widely used alcohol with boiling temperature $78.4^{\circ} \mathrm{C}$ and matrix-compatibility, as the active fluid (Supplementary Fig. 1 and Supplementary Discussion for a discussion of the material components choice).

Ethanol, included inside tiny micro-bubbles embedded in the elastic silicone rubber matrix, boils upon reaching the liquid-gas a

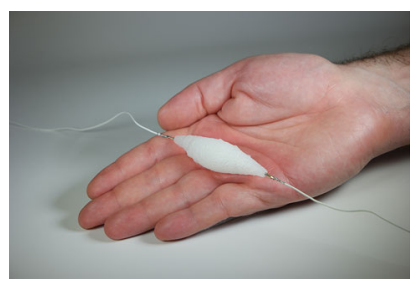

b

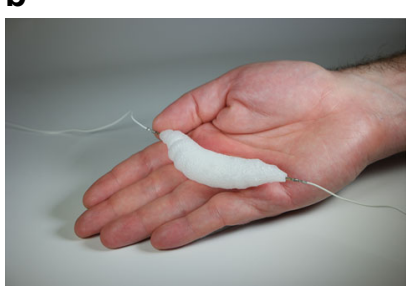

Fig. 1 Soft artificial muscle. The muscle is composed of ethanol distributed throughout the solid silicone elastomer matrix. a Electrically actuated muscle including thin resistive wire in a rest position on a human hand. $\mathbf{b}$ Expanded muscle actuated ( $8 \mathrm{~V}, 1 \mathrm{~A})$

transition temperature, accompanied by tremendous increase in volume, leading to significant expansion of the whole soft composite material. This composite material may be quickly and easily prepared by mixing ethanol with silicone elastomer (Supplementary Movie 1, Supplementary Software 1). The mixed material is both castable and 3D-printable (Supplementary Movies 2, 4), and after preparation will solidify in roomtemperature curing. We successfully mixed various amounts of ethanol (0-33 vol\%) in the two-part platinum-catalyzed silicone elastomer (Supplementary Fig. 2). In total 20 vol\% ethanol was chosen as optimal composition.

We show the material as an artificial muscle that can be electrically actuated using a thin resistive wire (Fig. 1a) and low power characteristics ( $8 \mathrm{~V}, 1 \mathrm{~A})$ to exhibit significant expansioncontraction ability (Fig. 1b).

Due to mixing, ethanol is distributed throughout the silicone elastomer matrix in bubble-shaped pores and forms local pressure equilibrium with its vapors. During curing, ethanol vapors occupy air pockets distributed throughout the material, create new pores and lower the developing internal vapor pressure by expanding the pores until the equilibrium with the external environment pressure is achieved. Ethanol wets both the silicone elastomer gel and the cured solid (Supplementary Fig. 1). Thus, ethanol spreads on the inner walls of the bubbles and the remainder of the space inside them, if any, is occupied by ethanol vapors and air (Fig. 2a). Density of the material including $20 \mathrm{vol} \%$ ethanol was measured at $0.84 \mathrm{~g} \mathrm{~cm}^{-3}$. Upon heating the composite to a temperature of $78.4^{\circ} \mathrm{C}$, ethanol boils and the local pressure inside the bubbles grows, forcing the elastic silicone elastomer matrix to comply by expansion in order to reduce the pressure (Fig. 2b).

Micro-CT scans of the composite material cross-section before and after activation illustrate the expansion phenomena and provide insight into the material at room temperature and during the heating with a spiral-shaped resistive wire (Fig. 2c). To facilitate the interpretation of the micro-CT scans, the material specimens were placed on top of a plastic container with liquid ethanol.

Liquid ethanol evaporates with temperature, giving rise to internal pressure inside the bubbles, which results in slightly expanded silicone elastomer matrix. When ethanol passes the liquid-vapor phase transition, extreme volume change occurs and the silicone elastomer matrix significantly expands. With growth in local pressure, the boiling temperature increases and thus, continued heating to temperatures slightly higher than $78.4^{\circ} \mathrm{C}$ is required for further expansion, until no liquid ethanol remains in the bubbles (Fig. 2b). Infrared radiation images of the material at room temperature and during expansion (using $\mathrm{Ni}-\mathrm{Cr}$ spiral) are shown in Fig. 2d.

Mechanical properties. A maximal volume expansion of about $915 \%$ was measured at the temperature of $90^{\circ} \mathrm{C}$ during controlled 
a

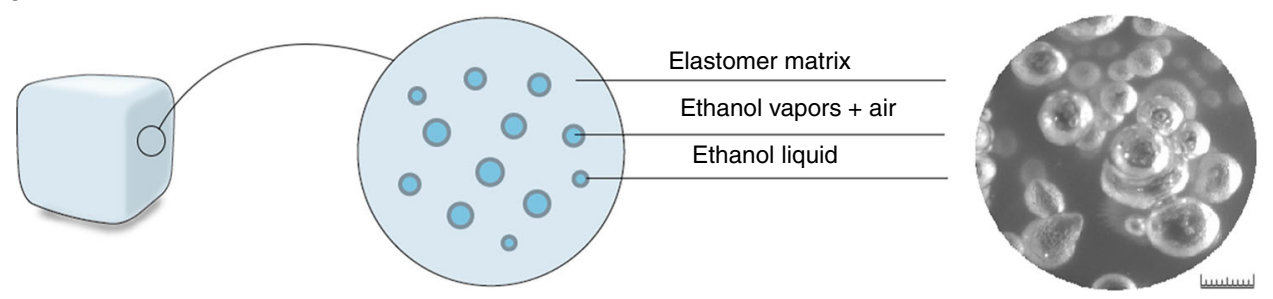

b
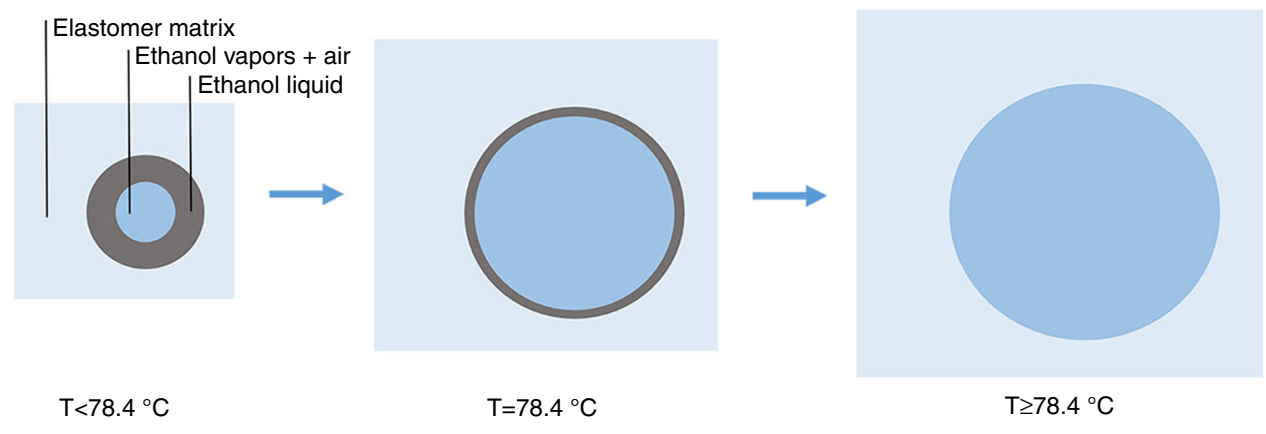

$\mathrm{T} \geq 78.4^{\circ} \mathrm{C}$

C

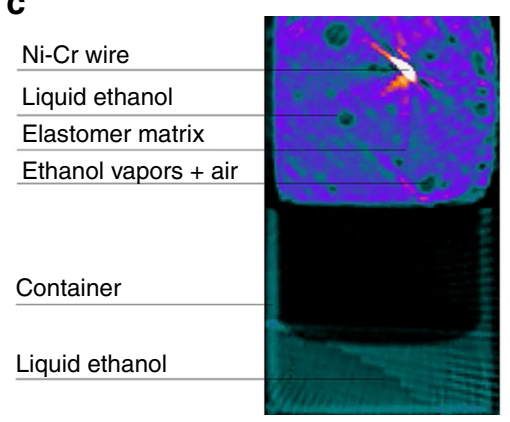

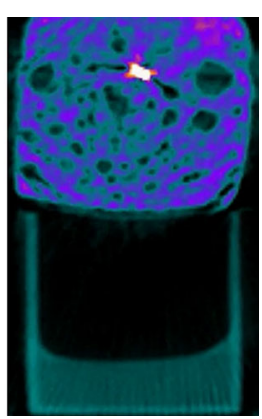

d

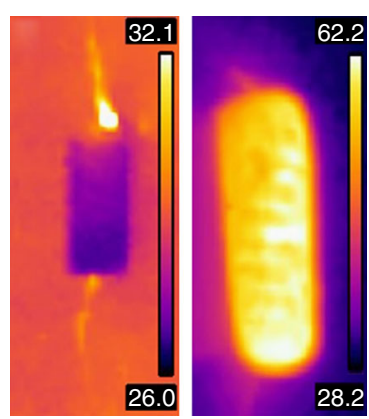

Fig. 2 Structure and principle of operation of the soft composite material. a Microstructure: Illustration and a stereoscope image; scale bar is $1 \mathrm{~mm}$. b Illustration of the expansion process on example of a single ethanol bubble. c Micro-CT images of the material cross-section at room temperature and during heating. $\mathbf{d}$ Infra-red images of the material as the heating starts and during expansion; the material is heated using $\mathrm{Ni}-\mathrm{Cr}$ resistive wire

heating in a wide water bath (unconstrained volumetric expansion). We used an Instron machine to measure the blocked directional force and actuation stress characteristics of the material during its electrical actuation using resistive spiral-shaped wire at low power $(15 \mathrm{~V}, 1 \mathrm{~A})$. First, we aimed to demonstrate an ability of the composite to lift a weight 1000 times more than its own. The $6 \mathrm{~g}$ material showed an ability of repeated lifting of a weight of $6.1 \mathrm{~kg}$, shown in Fig. $3 \mathrm{a}$ as 30 repeated cycles of loading to $60 \mathrm{~N}$ (blocked one-directional force). A detailed view of three loading-unloading cycles is shown in Fig. 3b. In a separate experiment, we measured maximal unidirectional force obtained at various blocked-strain levels in the $0-100 \%$ range. The maximal force for unstrained specimen weighing $2 \mathrm{~g}$ was about $120 \mathrm{~N}$, which is equivalent to the actuation stress of 1.3 $\mathrm{MPa}$ and a maximal ability of lifting weight 6000 times larger than its own. Figure $3 \mathrm{c}$ shows that the force decreases with strain down to $35 \mathrm{~N}$ at $100 \%$ blocked strain. An extrapolation of the trend suggests that the material will reach strain limit of $140 \%$, when allowed to expand in only one direction, as opposed to about $900 \%$ volumetric expansion when unconstrained. Both 3Dprinted and cast specimens show similar actuation behavior (Supplementary Movie 4).
Implementation in robotics. We demonstrate the implementation of our composite material as an actuator in a variety of robotic applications (Supplementary Movie 2). First, we show a McKibben-type muscle. Our self-sufficient artificial muscle does not require any compressors or pressure-regulating equipment (Fig. 4a), and is capable of lifting weight much larger than its own (for example, a $13 \mathrm{~g}$ actuator lifts $1 \mathrm{~kg}$ in Fig. 4b). We demonstrate its use as a bicep, which contracts and pulls the lower arm up, causing it to bend at the elbow (Fig. 4c). The actuator is comprised of the composite material placed inside a braided mesh sleeving, fixed at the edges (Supplementary Fig. 3). The actuation is electrically driven using a spiral-shaped resistive wire (powered at $30 \mathrm{~V}, 1.5 \mathrm{~A}$ ) passing inside the actuator. During the actuation, the composite material expands radially and contacts longitudinally, mimicking natural muscle behavior.

In addition, we designed an actuator consisting of our composite material attached to a layer of pure (unactuated) silicone elastomer, to create bimorph bending (Fig. 4d). The actuation is electrically driven using a resistive wire powered at 8 $\mathrm{W}(8 \mathrm{~V}, 1 \mathrm{~A})$. We used this actuator in two models of robots: the all-soft two-leg "worm" (Fig. 4e), and a "sleigh" (Fig. 4f). During the actuation, the composite material expands and bends due to 
a

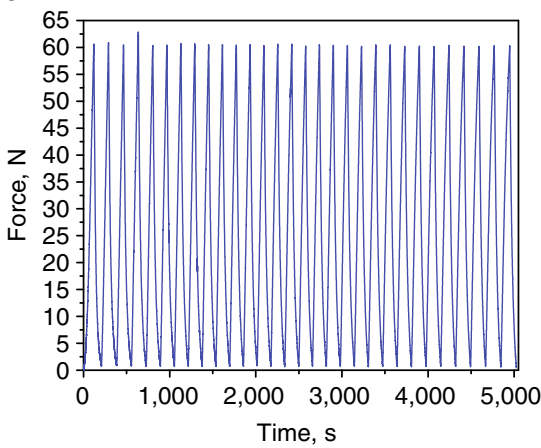

C

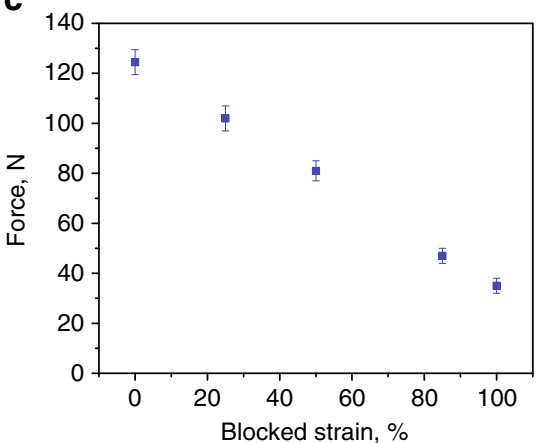

b

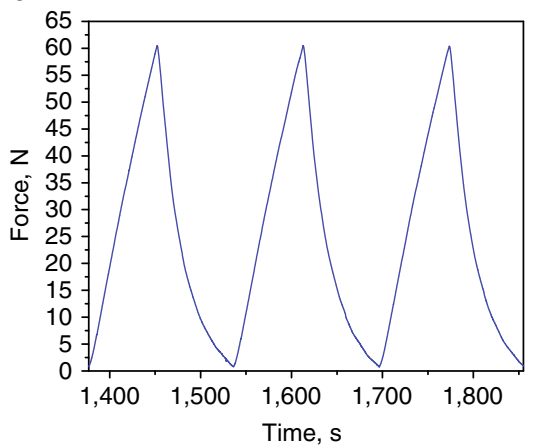

Fig. 3 Force-strain characteristics of the material (15 V, 1 A). a 30 following cycles of loading to $60 \mathrm{~N}$ (blocked force; cylindrical specimen; diameter 15.1 $\mathrm{mm}$, length $40 \mathrm{~mm}$; weight $6 \mathrm{~g}$ ). b Detailed view of three cycles in a. c Blocked force at various elongations for constrained cylindrical actuation; error bar relates to s.d. Specimen diameter $11.1 \mathrm{~mm}$, length $25 \mathrm{~mm}$; weight $2 \mathrm{~g}$. Note that the actuation depends on heating and cooling rates

the constricting force at the vicinity of the passive silicone elastomer layer.

Figure 4i shows an electrically driven soft gripper, comprised of the composite material with two soft legs at the edges for grasping. Upon actuation, bimorph bending occurs and the legs move towards the item (e.g., a raw egg) and enable its grasping and lifting.

In addition to soft body locomotion and grasping, we demonstrate the ability of the artificial muscle to substitute electrical motor in an existing robot. We used a biomimetic robot produced using evolutionary algorithms ${ }^{34}$. The original $\operatorname{robot}^{34}$ (Fig. 4g) was 3D-printed and contained a small electrical stepper motor embedded in the upper bar. We reprinted the original robot and replaced the electrical motor with a detachable 3D-printed unit (Supplementary Fig. 4) including our composite material as the active material (Fig. 4h) embedded in a Teflon sleeve. We applied an electrical current $(8 \mathrm{~V}, 1 \mathrm{~A})$ through a resistive coil embedded inside the muscle, which exhibited directional expansion and acted as a piston moving the upper part of the bar forward. We demonstrate the locomotion of each robot in Supplementary Movie 2.

\section{Discussion}

The proposed soft composite material demonstrates a combination of high strain (up to $>900 \%$ ) and correspondingly high stress (up to $1.3 \mathrm{MPa}$ ) at low density $\left(0.84 \mathrm{~g} \mathrm{~cm}^{-3}\right)$. Even at $100 \%$ strain the material develops stress of $0.4 \mathrm{MPa}$ and is capable of lifting weight about 1700 times greater than its own. These characteristics place this material in previously inaccessible region of the actuator stress-strain charts (Fig. 5a). Our actuators are Pareto-undominated in specific actuation stress versus strain (Fig. 5b). We suggest that the strain limit of our material is the maximal strain of the silicone elastomer matrix (980\%, according to the manufacturer). Along with its extremely low cost (laboratory cost of about 3 cent per gram), ease of fabrication, and environmental friendliness, these properties make this material an attractive solution where strain density is a critical factor.

Efficiency of the actuator heated by a resistive wire may be estimated as a ratio between the mechanical work produced per time, and the invested (consumed) electrical energy. Using data from Fig. 3, the consumed electrical power is a product of the applied DC voltage and current: $15 \mathrm{~V} \cdot 1 \mathrm{~A}=15 \mathrm{~W}$. According to Fig. 3b, the time it takes the actuator to reach a force of $60 \mathrm{~N}$ was 70 s. The strain level at $60 \mathrm{~N}$ force may be estimated from Fig. $3 \mathrm{c}$ as 70\%. Accordingly, linear expansion of the $40 \mathrm{~mm}$ long specimen was $0.7 \times 40 \mathrm{~mm}=28 \mathrm{~mm}=0.028 \mathrm{~m}$. Thus, the mechanical work done by the actuator may be calculated as a product of the force and the distance, namely $60 \mathrm{~N} \cdot 0.028 \mathrm{~m}=1.68 \mathrm{~J}$. The output power is $1.68 \mathrm{~J} / 70 \mathrm{~s}=0.024 \mathrm{~W}$. Thus, the efficiency of the actuator is $(0.024$ $\mathrm{W} / 15 \mathrm{~W}) \cdot 100 \% \approx 0.2 \%$. This value corresponds to heating caused by a single-coil of the resistive wire. In Supplementary Discussion and Supplementary Fig. 5 we show that using a wire of the same resistance in different designs (single-, double-, and triple-coiled wire), heating times may vary significantly. For instance, using triple-coiled wire shortens the heating time by $40 \%$, allowing to increase the efficiency by this value. In this way, a simple change in a wire design, allowing more uniform distribution of the heat, may increase the efficiency of our material to about $0.3 \%$. This value is comparable to the values for other thermal expansion actuators and for some shape memory alloys in the Ashby chart ${ }^{35}$. In Fig. 6 we show the efficiency plotted against the actuation strain for most of the existing actuation methods relevant for soft actuation, including materials (SMA, piezoelectric materials, etc) and devices (hydraulic and pneumatic setups).

It may be seen that actuation methods exhibiting high actuation strain (about 100\%) along with high efficiency are based on either hydraulic or pneumatic (FEA, about 87\%; PAM, about $40 \%$ ) devices. For DEAs ${ }^{36}$, depending on the mode of operation, 

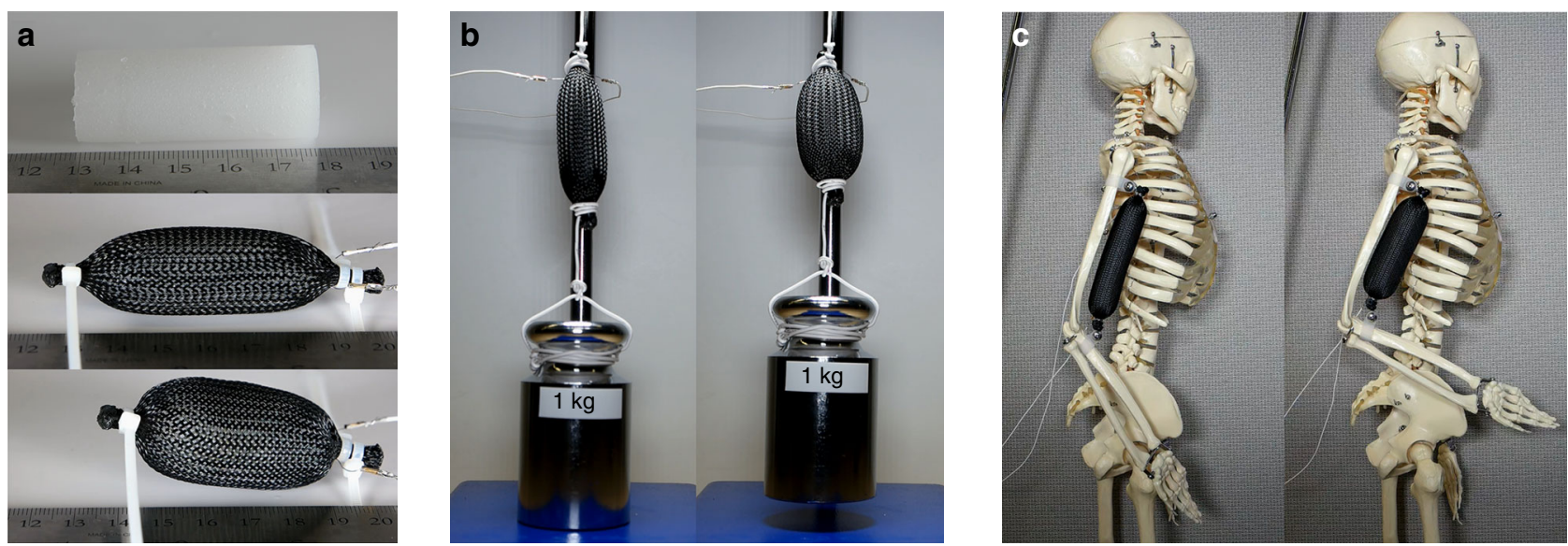

d
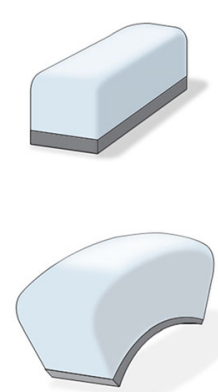

$\mathrm{Ni}-\mathrm{Cr}$ wire

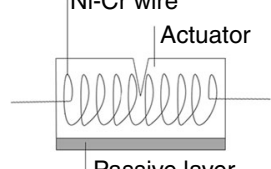

Passive layer
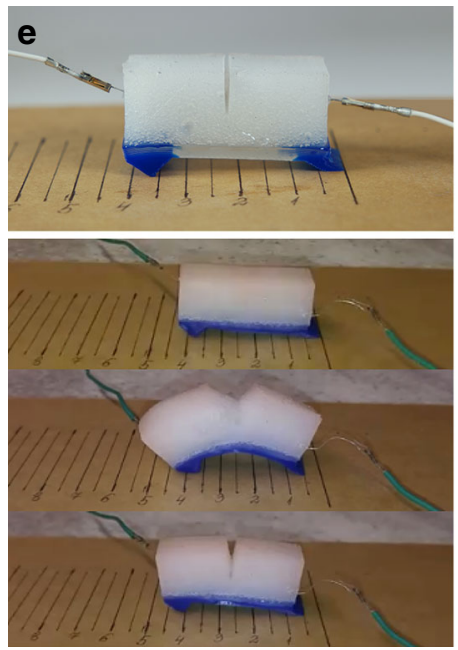
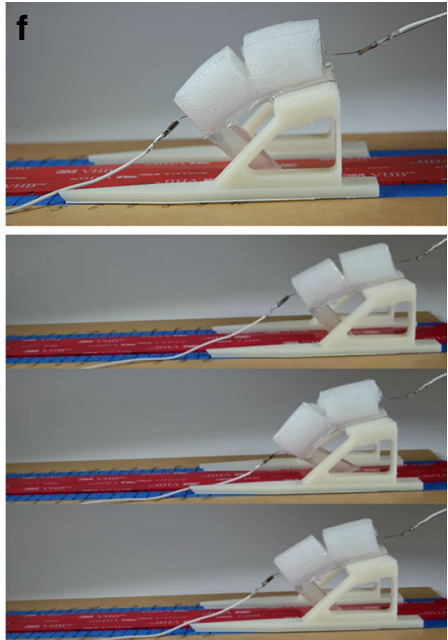
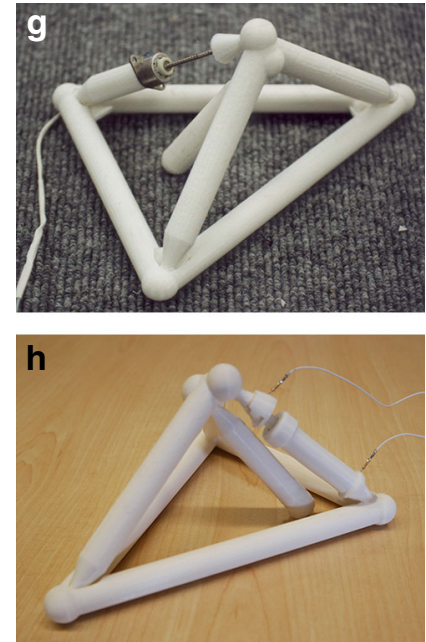

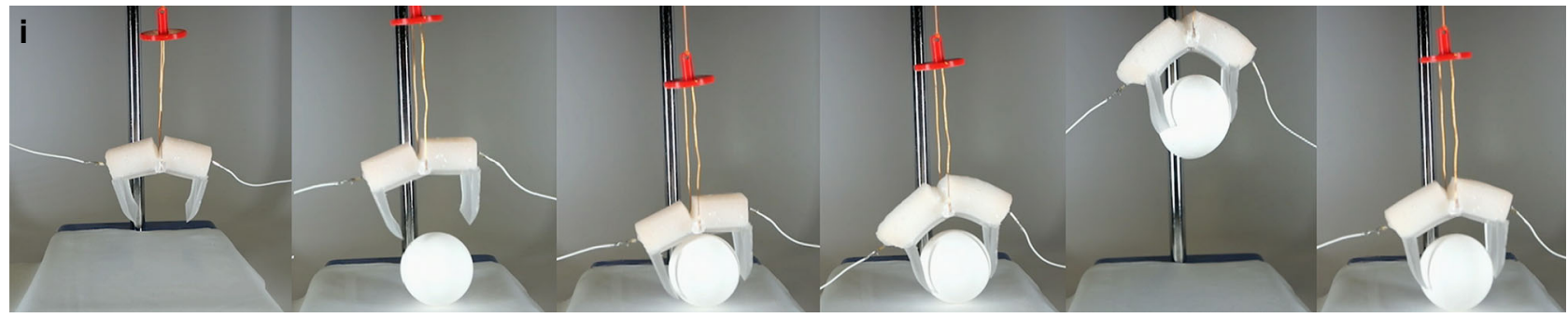

Fig. 4 Implementation of the soft composite material as an actuator. a McKibben-type artificial muscle (soft composite material inside braided mesh sleeving) shows displacement of about 25\%. b $13 \mathrm{~g}$ artificial muscle lifts the weight of $1 \mathrm{~kg}$. c Soft artificial muscle implemented as a biceps lifting skeleton's arm to $90^{\circ}$ position at elbow (a-c: actuation powered at $45 \mathrm{~W}$ (30 V, $1.5 \mathrm{~A}$ )). d Design of the bi-morph bending actuator. e All-soft two-leg "worm" and its locomotion powered at $8 \mathrm{~W}(8 \mathrm{~V}, 1 \mathrm{~A})$. $\mathbf{f}$ The sleigh robot and its locomotion powered at $8 \mathrm{~W}(8 \mathrm{~V}, 1 \mathrm{~A})$. $\mathbf{g}$ Tetrahedral robot evolved and $3 \mathrm{D}$-printed in $2000^{34}$ with embedded electrical motor. $\mathbf{h}$ The same robot with the soft composite material as an actuator embedded instead of the electrical motor. $\mathbf{i}$ Soft gripper lifting an egg (sequence from left to right; $8 \mathrm{~V}, 1 \mathrm{~A}$ )

the maximal efficiency value of $26 \%$ was reported for the acrylic actuators (which are also superior to other DEAs in strain). However, such hydraulic and pneumatic solutions require external compressors and pressure-regulating equipment. In turn, DEAs require very high voltage for their operation. According to the chart, the next possible solution is SMAs; however, they show efficiency of about $2 \%$ and strains $<10 \%$. This dichotomy highlights the state-of-the-art trade-off, in which a combination of reasonable efficiency and strain cannot be achieved using easilyoperated material-actuators based on low voltage, but only by devices requiring external system for power conversion (a compressor or high-voltage converter). In contrast, the proposed material offers high strain directly from low-voltage source, typically available in untethered applications. We suggest that the high actuation strain, along with low cost and simplicity of preparation of the proposed soft material, merit further exploration of methods to improve its efficiency, which would then allow efficient material-actuator with very high strain.

Efficiency and operation of the proposed actuator material highly depends on heating and cooling rates. For resistive heating, higher current or more distributed heating networks are likely to provide faster material expansion. For cooling, an optimized design of the actuator geometry and the surface area may facilitate faster cooling rates. For example, a thin strip with large surface-to-volume ratio is likely to cool much faster than bulk material. In addition, active cooling solutions such as a Peltier 
a

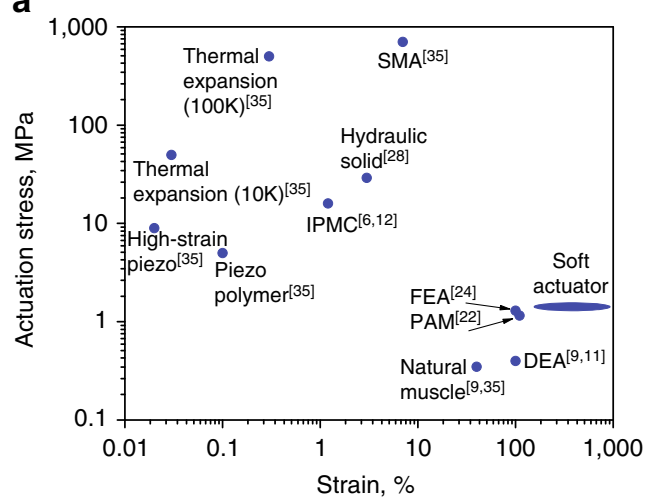

b

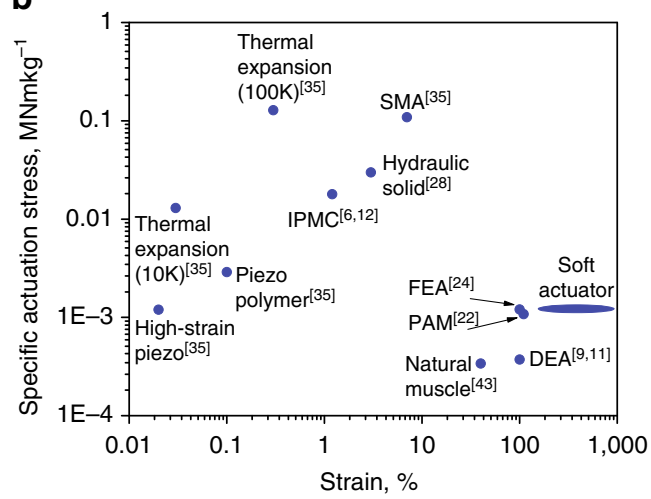

Fig. 5 Comparative stress-strain charts for electrically driven actuators. a Actuation stress plotted against strain. b Specific actuation stress (actuation stress divided by density of the material) plotted against strain. Abbreviations: DEA- dielectric elastomer actuator, FEA- fluidic elastomer actuator, IPMCionic polymer-metal composite, PAM- pneumatic artificial muscle (McKibben actuator), SMA- shape memory alloy. The proposed material is labeled "Soft Actuator". The ellipse designates a range of observed strains spanning from constrained unidirectional expansion of $140 \%$ to unconstrained volumetric expansion of $900 \%$. For Thermal Expansion actuators, 10 and $100 \mathrm{~K}$ are the temperature change ranges in Kelvin degrees

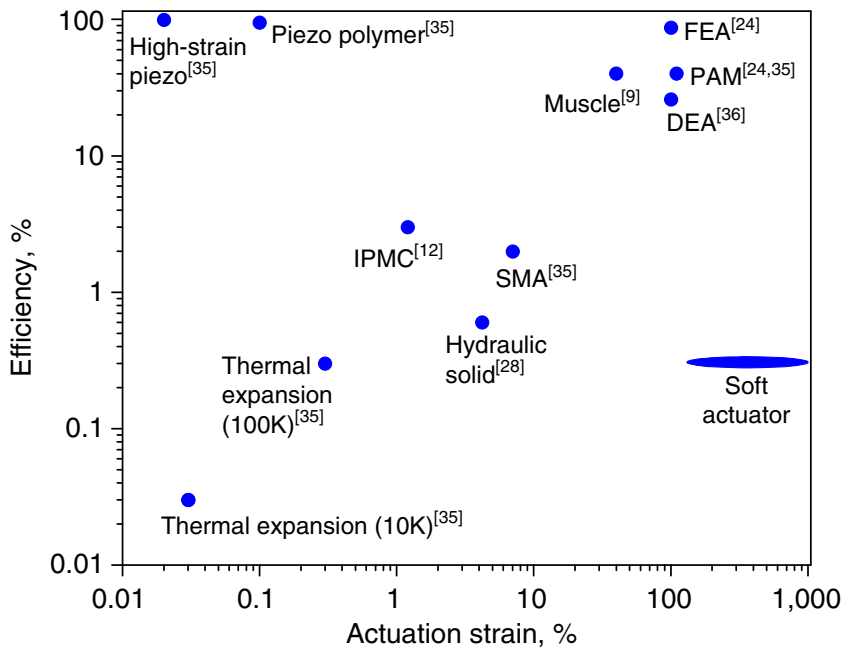

Fig. 6 Maximal efficiency plotted versus actuation strain for various actuating methods. Our material is labeled as "Soft Actuator". For Thermal Expansion actuators, 10 and $100 \mathrm{~K}$ are the temperature change ranges in Kelvin degrees. The elliptical shape denotes the range of strain possible ranging from purely linear expansion (140\%) to full (unconstrained) volumetric expansion (900\%)

Junction, or a liquid-flow cooling channels could also be used. In the latter, as silicone repels water (Supplementary Fig. 1), internal channels may be designed in the soft material for water-flow cooling. However, such solutions would require additional power and space on the potential robot/device.

Alternatively, we suggest a biology inspired solution using agonist-antagonist muscle pairs. It is well known that during the contraction of biceps to bend the arm at the elbow, triceps are relaxed, and vice versa. This feature may be used to significantly reduce the de-actuation time of the proposed actuator. Once one actuator (biceps) will complete its work to bend the arm, the task of bringing the arm back into its initial open position may be done by triggering the second actuator (triceps), instead of waiting for the cooling of the biceps (Fig. 7). In the Supplementary Movie 3 we show that this method may increase the actuation/de-actuation time 2.4 times.
To summarize, our work proposed a self-contained soft robust composite material, combining very high strain and reasonably high stress with low density, which is easily produced from bio-compatible components at a very low cost. This materialactuator may serve in a variety of applications, from traditional robotics to advanced bio-medical needs, and may enable a new kind of entirely soft robots.

\section{Methods}

Materials. We used platinum-catalyzed two-part silicone rubber Ecoflex 00-50 (Smooth-On, PA, USA) as a matrix material and ethanol $\geq 99.5 \%$ (Sigma Aldrich, MO, USA) as an active phase change material. Properties of the silicone rubber are shown in Table 1 below. Material preparation involves thorough hand-mixing of $20 \mathrm{vol} \%$ of ethanol with silicone elastomer (first with part A for about $2 \mathrm{~min}$, then mixed with part B for about $2 \mathrm{~min}$ ). The material is ready-to-cast and ready-toprint after the preparation. Room temperature curing of the cast or 3D-printed part takes up to $3 \mathrm{~h}$. A commercially available $0.25 \mathrm{~mm}$ diamter Ni-chrome resistive wire was used for electrically driven heating of the artificial muscle (i.e., for the actuation). To comply with the expansion of the actuator material, a helical spiral shape was chosen for the $\mathrm{Ni}-\mathrm{Cr}$ wire. The wire was hand-wound on an $8 \mathrm{~mm}$ screw driver shaft as shown in Supplementary Fig. 6.

3D-Printing. Fabrication of the actuator using 3D-Printer was performed on a lab-made desktop 3D-printer capable of direct printing of two materials in one print. For optimal printing using 14 gauge syringe tip, the material was held in the mixing container for $15 \mathrm{~min}$ before printing. The maximum 3D printing resolution of $0.8 \mathrm{~mm}$ was achieved using 20 gauge syringe tip.

$3 \mathrm{D}$-printing of the robotic demonstrators was done on commercial FDM machines: Ultimaker 2+, Ultimaker (Gendermalsen, Netherlands) for the sleigh robot (material: PLA), and uPrint, Stratasys (MN, USA) for tetragonal evolved robot (material: acrylonitrile butadiene styrene (ABS)).

Characterization. Olympus SZ51 (Tokyo, Japan) stereoscope with analytic software was used for optical characterization of the actuator material. We used $30 \times$ $10 \times 3 \mathrm{~mm}$ size specimens and appropriate lighting conditions to obtain images with highest contrast. Micrometrix AccyPyc II1340 (GA, USA) pycnometer was used for density evaluation of the actuator material. Ramé-hart (NJ, USA) model 190CA goniometer was used for contact angle measurements. Headspace Gas Chromatography Mass Spectrometry (GC-MS) analysis was performed on the PerkinElmer Clarus SQ8C model (Waltham, MA, USA) apparatus. Micro-CT (Mediso, Boston, MA), a small-animal CT scanner with an image resolution of 100 microns, was used for $\mathrm{x}$-ray computer tomography (CT) of the internal structure of the actuator material. Water bath experiment was conducted for evaluation of the maximal expansion of the actuator material. A desktop hot-plate was used to heat the water bath (borosilicate glass beaker) while the water temperature was continuously controlled by a K-type thermocouple connected to the Fluke 52-2 dual input digital thermometer $\left( \pm 0.1^{\circ} \mathrm{C}\right)$. The water level was monitored during the experiment and the volume was constantly evaluated. An Instron 8841 (MA, USA) machine was used for mechanical properties evaluation. For the blocked force experiment, the cylindrical muscle specimen $(40 \mathrm{~mm}$ length, $15.1 \mathrm{~mm}$ diameter) was placed in a polytetrafluoroethylene (PTFE) cylinder, and inserted into a hollow 
a

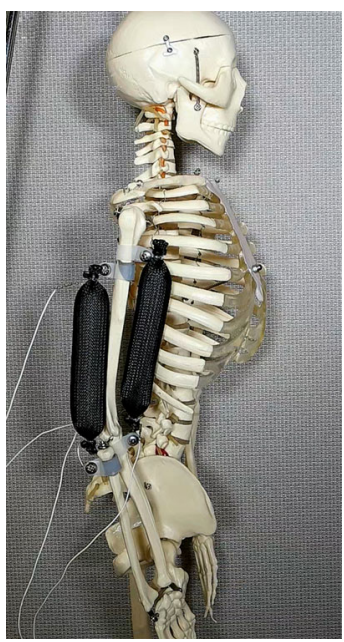

b

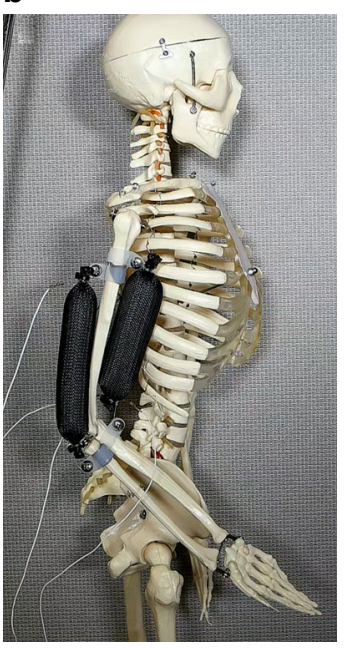

C

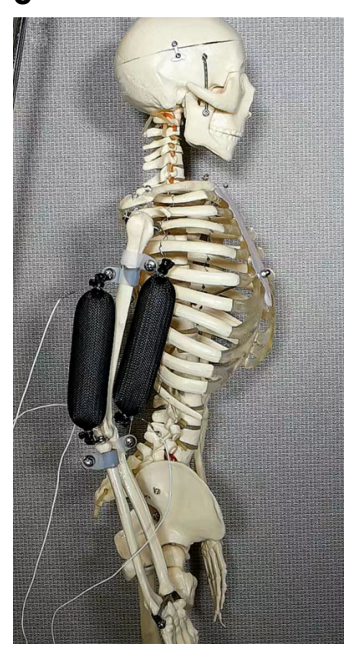

Fig. 7 Agonist-antagonist soft actuator pair ( $20 \mathrm{~V}, 1 \mathrm{~A}$ ). a Initial position of biceps and triceps actuators; b Actuation (bending the arm) by biceps; c De-actuation (bringing the arm to its initial position) by triceps. Actuators size: $20 \mathrm{~mm}$ diameter, $100 \mathrm{~mm}$ length. This setup reduced actuation time by a factor of 2.4 compared with a single actuator

Table 1 Properties of the silicone rubber Ecoflex 00-50 (manufacturer declared)

\begin{tabular}{|c|c|c|c|c|c|c|c|}
\hline Ecoflex 00-50 & $1.07 \mathrm{~g} \mathrm{~cm}^{-3}$ & $8000 \mathrm{cps}$ & $18 \min$ & $3 \mathrm{~h}$ & $00-50$ & $315 \mathrm{psi}$ & $980 \%$ \\
\hline
\end{tabular}

aluminum cylinder with a closed top. The latter was attached to the upper static part of the Instron machine, while the specimen at the bottom faced a smaller aluminum cylinder connected directly to the load cell. The muscle was actuated by heating, provided using a $\mathrm{Ni}-\mathrm{Cr}$ resistive wire and low power $(15 \mathrm{~V}, 1 \mathrm{~A})$. At heating, the muscle expanded and pressed against the bottom cylinder connected to the load cell, which detected and recorded the force. The experiment setup schematic is shown in Supplementary Fig. 7. Displacement against force was measured in a similar experiment, at which specimens with constant dimensions $(25 \mathrm{~mm}$ length, $11.1 \mathrm{~mm}$ diameter) were placed in the PTFE tubes with various dimensions to enable expansion to a desired strain levels. Then blocked force was measured at the developed strain. Five specimens were tested in each testing set. The volume expansion and stress-strain experiments were conducted $24 \mathrm{~h}$ after the curing of the specimens.

Data availability. All data are available from the authors upon reasonable request.

Received: 25 April 2017 Accepted: 16 July 2017

Published online: 19 September 2017

\section{References}

1. Rus, D. \& Tolley, M. T. Design, fabrication and control of soft robots. Nature 521, 467-475 (2015).

2. Laschi, C. \& Cianchetti, M. Soft robotics: new perspectives for robot bodyware and control. Front. Bioeng. Biotechnol. 2, 3 (2014).

3. Kim, S., Laschi, C. \& Trimmer, B. Soft robotics: a bioinspired evolution in robotics. Trends Biotechnol. 31, 287-294 (2013).

4. Trimmer, B. Soft robots. Curr. Biol. 23, R639-R641 (2013).

5. Trivedi, D., Rahn, C. D., Kier, W. M. \& Walker, I. D. Soft robotics: biological inspiration, state of the art, and future research. Appl. Bionics Biomech. 5, 99-117 (2008)

6. Kim, K. J. \& Shahinpoor, M. A novel method of manufacturing threedimensional ionic polymer-metal composites (IPMCs) biomimetic sensors, actuators and artificial muscles. Polymer 43, 797-802 (2002).

7. Cianchetti, M., Mattoli, V., Mazzolai, B., Laschi, C. \& Dario, P. A new design methodology of electrostrictive actuators for bio-inspired robotics. Sensors Actuators B Chem. 142, 288-297 (2009).
8. O'Halloran, A., O'Malley, F. \& McHugh, P. A review on dielectric elastomer actuators, technology, applications, and challenges. J. Appl. Phys. 104, 71101 (2008).

9. Mirfakhrai, T., Madden, J. D. W. \& Baughman, R. H. Polymer artificial muscles. Mater. Today 10, 30-38 (2007).

10. Bar-Cohen, Y. EAP as artificial muscles - progress and challenges. inSmart Structures and Materials (ed. Bar-Cohen, Y.) 10-16 (International Society for Optics and Photonics, 2004). doi:10.1117/12.538698

11. Galantini, F., Carpi, F. \& Gallone, G. Effects of plasticization of a soft silicone for dielectric elastomer actuation. Smart Mater. Struct. 22, 104020 (2013)

12. Shahinpoor, M. \& Kim, K. J. Ionic polymer-metal composites: I. Fundamentals. Smart Mater. Struct. 10, 819-833 (2001).

13. Huang, W. M. et al. Shape memory materials. Mater. Today 13, 54-61 (2010).

14. Ratna, D. \& Karger-Kocsis, J. Recent advances in shape memory polymers and composites: a review. J. Mater. Sci. 43, 254-269 (2007).

15. Laschi, C. et al. Soft Robot Arm Inspired by the Octopus. Adv. Robot. 26, 709-727 (2012).

16. De Greef, A., Lambert, P. \& Delchambre, A. Towards flexible medical instruments: Review of flexible fluidic actuators. Precis. Eng. 33, 311-321 (2009).

17. Paik, J. Characterization of silicone rubber based soft pneumatic actuators. inIEEE/RSJ International Conference on Intelligent Robots and Systems4446-4453 (IEEE, 2013). doi:10.1109/IROS.2013.6696995

18. Polygerinos, P., Wang, Z., Galloway, K. C., Wood, R. J. \& Walsh, C. J. Soft robotic glove for combined assistance and at-home rehabilitation. Rob. Auton. Syst. 73, 135-143 (2015).

19. Shepherd, R. F. et al. Multigait soft robot. Proc. Natl Acad. Sci 108, 20400-20403 (2011).

20. Tolley, M. T. et al. A resilient, untethered soft robot. Soft Robot 1, 213-223 (2014).

21. Marchese, A. D., Katzschmann, R. K. \& Rus, D. A recipe for soft fluidic elastomer robots. Soft Robot 2, 7-25 (2015).

22. Chou, C.-P. \& Hannaford, B. Measurement and modeling of McKibben pneumatic artificial muscles. IEEE Trans. Robot. Autom 12, 90-102 (1996).

23. Morin, S. A. et al. Camouflage and display for soft machines. Science 337, 828-832 (2012).

24. Sridar, S. et al. Hydro Muscle -a novel soft fluidic actuator. In IEEE International Conference on Robotics and Automation (ICRA) 4014-4021 (IEEE, 2016). 
25. Bartlett, N. W. et al. A 3D-printed, functionally graded soft robot powered by combustion. Science 349, 161-165 (2015).

26. Ogden, S., Klintberg, L., Thornell, G., Hjort, K. \& Bodén, R. Review on miniaturized paraffin phase change actuators, valves, and pumps. Microfluid. Nanofluidics 17, 53-71 (2014).

27. Carlen, E. T. \& Mastrangelo, C. H. Electrothermally activated paraffin microactuators. J. Microelectromech. Syst. 11, 165-174 (2002).

28. Lipton, J. I., Angle, S., Banai, R. E., Peretz, E. \& Lipson, H. Electrically actuated hydraulic solids. Adv. Eng. Mater. 18, 1710-1715 (2016).

29. Konishi, S., Kawai, F. \& Cusin, P. Thin flexible end-effector using pneumatic balloon actuator. Sens. Actuat. A Phys 89, 28-35 (2001).

30. Ma, M., Guo, L., Anderson, D. G. \& Langer, R. Bio-inspired polymer composite actuator and generator driven by water gradients. Science 339, 186-189 (2013).

31. Zhao, Q. et al. An instant multi-responsive porous polymer actuator driven by solvent molecule sorption. Nat. Commun. 5, 4293 (2014).

32. Zhou, Z., Li, Q., Chen, L., Liu, C. \& Fan, S. A large-deformation phase transition electrothermal actuator based on carbon nanotube-elastomer composites. J. Mater. Chem. B 4, 1228-1234 (2016).

33. Altmüller, R., Schwödiauer, R., Kaltseis, R., Bauer, S. \& Graz, I. M. Large area expansion of a soft dielectric membrane triggered by a liquid gaseous phase change. Appl. Phys. A 105, 1-3 (2011).

34. Pollack, J. B. \& Lipson, H. Automatic design and manufacture of robotic lifeforms. Nature 406, 974-978 (2000).

35. Huber, J. E., Fleck, N. A. \& Ashby, M. F. The selection of mechanical actuators based on performance indices. Proc. R. Soc. A Math. Phys. Eng. Sci. 453, 2185-2205 (1997).

36. Bigue, J.-P. L. \& Plante, J.-S. Experimental study of dielectric elastomer actuator energy conversion efficiency. IEEE/ASME Trans. Mechatron. 18, 169-177 (2013).

\section{Acknowledgements}

We wish to acknowledge the help of the Columbia University Preclinical Molecular Imaging Laboratory under the direction of Dr. Lynne Johnson and specifically the technical assistance of Ms. Krissy Rodriquez in performing the micro-CTs. The authors thank Mr. Artur Autz of the Department of Bio-Medical Engineering in Columbia University for providing the mechanical testing equipment, Prof. Ngai Yin Yip of the Department of Earth and Environmental Engineering in Columbia University for providing the goniometer for contact angle measurements, and Mr. Drim Stokhuijzen of Delft University, Netherlands for assistance with illustrations.

\section{Author contributions}

A.M. developed the concept, designed and executed all the experiments, analyzed all the data, composed the manuscript; K.S. took part in the preliminary experiments at the concept development stage; H.L. supervised the research, developed the concept, analyzed the data and composed the manuscript.

\section{Additional information}

Supplementary Information accompanies this paper at doi:10.1038/s41467-017-00685-3.

Competing interests: The authors declare no competing financial interests.

Reprints and permission information is available online at http://npg.nature.com/ reprintsandpermissions/

Publisher's note: Springer Nature remains neutral with regard to jurisdictional claims in published maps and institutional affiliations.

cc) (i) Open Access This article is licensed under a Creative Commons Attribution 4.0 International License, which permits use, sharing, adaptation, distribution and reproduction in any medium or format, as long as you give appropriate credit to the original author(s) and the source, provide a link to the Creative Commons license, and indicate if changes were made. The images or other third party material in this article are included in the article's Creative Commons license, unless indicated otherwise in a credit line to the material. If material is not included in the article's Creative Commons license and your intended use is not permitted by statutory regulation or exceeds the permitted use, you will need to obtain permission directly from the copyright holder. To view a copy of this license, visit http://creativecommons.org/ licenses/by/4.0/.

(C) The Author(s) 2017 